\title{
Postjunctional effect of pinacidil on contractility of isolated bovine trachealis
}

\author{
P. Song, D. Rocchi, M. Lazzarotti, E. Crimi, K. Rehder, V. Brusasco
}

Postjunctional effect of pinacidil on contractility of isolated bovine trachealis. P. Song, D. Rocchi, M. Lazzarotti, E. Crimi, K. Rehder, V. Brusasco. COERS Journals Ltd 1996. ABSTRACT: Potassium channel openers hyperpolarize the smooth muscle cell membrane and relax airway smooth muscle. In this study, pre- and postjunctional effects of pinacidil ( \pm ) N-cyano-N'-(4-pyridil)-N"-(1,2,2-trimethylpropyl)-guanidine monohydrated), an adenosine triphosphate (ATP)-sensitive $\mathrm{K}^{+}$-channel opener, were determined in isolated bovine trachealis.

The effects of pinacidil on the frequency-response to electrical field stimulation (EFS), 0.1-32 Hz, and on the concentration-response to acetylcholine ( $\mathrm{ACh}), \mathbf{1 0}^{-9}$ $10^{-4} \mathrm{M}$, were compared in muscle strips from six animals. In addition, the effect of pinacidil on the inhibitory nonadrenergic noncholinergic (iNANC) system was evaluated in histamine-contracted muscle strips from another eight animals.

Pinacidil $\left(10^{-6}\right.$ or $\left.10^{-5} \mathrm{M}\right)$ shifted both the EFS frequency-response and the $\mathrm{ACh}$ concentration-response curves significantly $(p<0.01)$ to the right. Glibenclamide $\left(10^{-7}-10^{-5} \mathrm{M}\right)$ antagonized these responses in a concentration-dependent manner. The inhibitory effects of pinacidil on contractions of the same magnitude induced by EFS or exogenous ACh were not significantly different $(p=0.11)$, suggesting that pinacidil had only a postjunctional effect. Pinacidil had no effect on iNANC-mediated muscle relaxation.

We conclude that pinacidil attenuates the contraction of isolated bovine tracheal smooth muscle by postjunctional mechanisms.

Eur Respir J., 1996, 9, 2057-2063.

In smooth muscle, potassium channel openers (KCOs) increase $\mathrm{K}^{+}$efflux across the cell membrane, resulting in cell membrane hyperpolarization, reduction of $\mathrm{Ca}^{2+}$ influx through $\mathrm{L}$-type voltage-dependent $\mathrm{Ca}^{2+}$-channels $[1,2]$, and relaxation. There is disagreement about whether the effects of KCOs occur directly at the muscle cell (postjunctional) or at the muscle cell and nerve level (pre- and postjunctional) [3-6]. For instance, in guinea-pig trachealis the adenosine triphosphate (ATP)sensitive $\mathrm{K}^{+}$-channel (KATP) opener, cromakalim, affects the excitatory nonadrenergic noncholinergic (eNANC) nervous system [4]. If the effect of the KCOs is predominantly on the eNANC nervous system, it may not be demonstrable in airway tissue from animals with no eNANC nerves, such as the human and bovine airways. In these species, the effect of KCOs would occur only through inhibition of excitatory cholinergic nerves, stimulation of inhibitory nonadrenergic noncholinergic (iNANC) nerves, or a direct effect on the muscle. Importantly, a direct effect on the muscle may be easier to demonstrate in airways from species without eNANC nerves.

In this study, we compared the attenuation of isometric contractions of isolated bovine trachealis induced by electrical field stimulation (EFS) or by exogenous acetylcholine (ACh) in the presence of pinacidil (PIN), a KATP opener. PIN had similar inhibitory effects on contractions induced by EFS or ACh, suggesting a postjunctional effect only.
Cattedra di Fisiopatologia Respiratoria, DISM, Facoltà di Medicina e Chirurgia, Università di Genova, Genova, Italy.

Correspondence: V. Brusasco Dipartimento di Scienze Motorie Facoltà di Medicina e Chirurgia Largo R. Benzi 10 16132 Genova Italy

Keywords: Acetylcholine airway smooth muscle electrical field stimulation excitatory nonadrenergic noncholinergic inhibitory nonadrenergic noncholinergic potassium channel opener

Received: January 81996 Accepted after revision June 191996

Supported in part by a grant from MURST, Rome, Italy.

\section{Methodology}

\section{General methods}

Bovine tracheae were obtained from the local abattoir. Immediately after the death of the animals, the lower middle portions of the tracheae were removed and immersed in aerated $\left(95 \% \mathrm{O}_{2}\right.$ and $5 \% \mathrm{CO}_{2}$ ) physiological salt solution (PSS) $\left(4^{\circ} \mathrm{C}\right)$ of the following composition (mM): $0.8 \mathrm{MgSO}_{4}, 1.2 \mathrm{KH}_{2} \mathrm{PO}_{4}, 3.4 \mathrm{KCl}, 2.4$ $\mathrm{CaCl}_{2}, 110.5 \mathrm{NaCl}, 25.7 \mathrm{NaHCO}_{3}$, and 5.6 dextrose. This tissue was transported to the laboratory within approximately $1 \mathrm{~h}$. The cartilage rings were cut on the ventral side, and the mucosa and adventitia [7] were mechanically removed while the tracheae were immersed in PSS. Muscle strips (approximately $10 \mathrm{~mm}$ long and 2-3 mm wide) were mounted in $25 \mathrm{~mL}$ water-jacketed glass tissue baths containing aerated PSS at $37^{\circ} \mathrm{C}$. Indomethacin $10^{-5} \mathrm{M}$ was added to the PSS to antagonize the effect of endogenously produced prostaglandins; the effect of incubation of muscle strips with $10^{-5} \mathrm{M}$ indomethacin was studied in 14 strips from five additional animals. The lower end of each strip was anchored to a stationary hook via a silk string and the upper end was connected by a silk string to a force transducer (Grass Medical Instruments, Model FT03D) mounted on a micromanipulator, allowing the length of the strips to be gradually increased. 
Muscle strips were contracted for $30 \mathrm{~s}$ at 5 min intervals by EFS ( $0.5 \mathrm{~ms}$ pulse duration, $25 \mathrm{~Hz}$, and $25 \mathrm{~V})$. EFS was provided by a DC amplifier (Mayo Clinic, Section of Engineering) triggered by an electric stimulator (Grass Medical Instruments, Model S44) via a pair of vertically mounted parallel platinum plate electrodes $(1 \times 4 \mathrm{~cm})$. The isometric force developed by the muscle was continuously recorded on a Gould TA4000 stripchart recorder. Over a $2 \mathrm{~h}$ period, the muscle was stretched after each stimulation until the contractions were constant and maximal (optimal length). During this procedure, the muscle was repeatedly washed with PSS containing $10^{-5} \mathrm{M}$ indomethacin. The optimal length was not altered throughout the study.

At the end of the study, all strips were blotted dry, the excess tissue removed and the strips were weighed.

\section{Experimental procedures}

Time course of the effect of PIN. To test the time course of the effect of PIN, one strip from each of seven animals was incubated with PIN $10^{-5} \mathrm{M}$, and another strip from each of the seven animals served as a control for the effect of time. EFS $(0.5 \mathrm{~ms}$ pulse duration, $25 \mathrm{~Hz}$, and $25 \mathrm{~V}$ ) was applied before the incubation with PIN and every $10 \mathrm{~min}$ for $120 \mathrm{~min}$ after addition of PIN. Five strips from five additional animals were incubated for $2 \mathrm{~h}$ with $10^{-5} \mathrm{M}$ PIN and were then washed every 5 min with PSS until the effect of PIN had been reversed.

Effect on EFS- and ACh-induced contractions. Eight strips were prepared from each of another six animals. For each strip, the contractile response to ACh $10^{-4} \mathrm{M}$ was first determined. This response was defined as maximal. The strips were then washed with PSS until the resting forces had been re-established. Thereafter, four strips were contracted with EFS $(0.1-32 \mathrm{~Hz})$ every 5 min (in random order). The other four strips were incubated for $20 \mathrm{~min}$ with tetrodotoxin (TTX) $10^{-6} \mathrm{M}$ and then contracted with cumulatively increasing concentrations of $\mathrm{ACh}\left(10^{-9}\right.$ to $10^{-4} \mathrm{M}$, half-log increments). After completing the frequency-response to EFS and the concentration-response to $\mathrm{ACh}$, all eight strips were washed with PSS until the resting forces were re-established.

Of the four strips previously contracted by EFS, one strip each was incubated for $1 \mathrm{~h}$ with PIN $10^{-7}, 10^{-6}$ or $10^{-5} \mathrm{M}$, whilst the fourth strip, not incubated with PIN, served as a control for the effect of time. The frequencyresponse to EFS was determined again. Of the four strips previously contracted by $\mathrm{ACh}$, one strip each was incubated for 40 min with PIN $10^{-7}, 10^{-6}$ or $10^{-5} \mathrm{M}$, whilst the fourth, not incubated with PIN, served as a control for the effect of time. After the incubation with PIN, the muscles were incubated with TTX $10^{-6} \mathrm{M}$ for $20 \mathrm{~min}$ and the concentration-response to $\mathrm{ACh}$ was repeated.

Effect of glibenclamide. Two strips from each of another four animals were first contracted with $\mathrm{ACh} 10^{-4} \mathrm{M}$ to determine maximal force. After wash-out, all strips were contracted by EFS $(0.5,2,8$ and $32 \mathrm{~Hz})$. One strip was then incubated for $1 \mathrm{~h}$ with PIN $10^{-6} \mathrm{M}$, while the other strip served as control for the effect of time. The frequency-response to EFS was then repeated. Glibenclamide $\left(10^{-7}, 10^{-6}\right.$, and $\left.10^{-5} \mathrm{M}\right)$ was added cumulatively to the strips incubated with PIN. After $30 \mathrm{~min}$ incubation with each of the three glibenclamide concentrations, the frequency-response to EFS was repeated.

Four strips from each of another four animals were first contracted with $\mathrm{ACh} 10^{-4} \mathrm{M}$ to determine maximal force. After wash-out, all strips were incubated for 20 min with TTX $10^{-6} \mathrm{M}$ and then contracted with cumulatively increasing concentrations of ACh $\left(10^{-7}\right.$ to $10^{-4} \mathrm{M}$, in $\log$ increments). After wash-out, three strips were incubated with PIN $10^{-6} \mathrm{M}$ for $1 \mathrm{~h}$ and TTX $10^{-6} \mathrm{M}$ for 20 min, while the fourth strip incubated with TTX but not with PIN served as control for the effect of time. The concentration-response to ACh was then repeated. After wash-out, the strips were again incubated with PIN $10^{-6} \mathrm{M}$, TTX $10^{-6} \mathrm{M}$, and glibenclamide $10^{-7}, 10^{-6}$ or $10^{-5} \mathrm{M}$. The concentration-response to $\mathrm{ACh}$ was then repeated.

Effect on iNANC. Eight pairs of strips from each of another eight animals were incubated with $10^{-6} \mathrm{M}$ propranolol and $10^{-5} \mathrm{M}$ atropine. One strip of each pair was additionally incubated for $1 \mathrm{~h}$ with $10^{-6} \mathrm{M}$ PIN. All eight strips were then contracted by histamine $10^{-4} \mathrm{M}$ and the frequency-response to EFS $(4,8,16,32$ and 64 $\mathrm{Hz}$ ) was determined in all eight strips.

\section{Data analysis}

All active forces were expressed as percentage of maximal forces. All forces observed after incubation with PIN were corrected for the effect of time using the following equation:

\section{Rcorrected $=$ Rbefore $($ Rafter/Rbefore - Cafter/Cbefore +1$)$}

were $\mathrm{R}$ indicates forces developed by PIN-incubated muscles and $\mathrm{C}$ forces of control muscles.

To determine the pre- and postjunctional effect of PIN, the concentration of ACh and the frequency of EFS resulting in 30 or $50 \%$ of the maximal response (ACh $10^{-4} \mathrm{M}$ ) were determined by linear interpolation on each of the frequency- and concentration-response curves obtained before incubation with PIN. Concentration and frequency-response curves were smoothed either by eye or cubic polynomial fitting. The response of the PINtreated muscles was then determined at the same ACh concentration and EFS frequency.

\section{Statistical analysis}

Two- and three-factor repeated-measures analysis of variance (ANOVA) were used for statistical analysis. The independent factors for comparisons between the effects of ACh and EFS were type of stimulus (ACh or EFS), amplitude of response (30 or $50 \%$ of maximal response), and concentration of PIN. P-values less than 0.05 were considered statistically significant. Results are presented as mean $\pm \mathrm{SD}$. 


\section{Drugs}

Pinacidil (( \pm ) N-cyano-N'-(4-pyridil)-N"-(1,2,2-trimethylpropyl)-guanidine monohydrated) was kindly provided as dry powder by Formenti S.p.A. (Milan, Italy). A $10^{-4} \mathrm{M}$ stock solution of PIN was prepared in acidified alcohol (35\% ethanol and $0.35 \% 10 \mathrm{~N} \mathrm{HCl}$, vol/vol) and diluted with distilled water immediately before each experiment. Tetrodotoxin (sodium citrate buffered) was purchased from ICN (Milan, Italy), and indomethacin crystalline, acetylcholine free base, histamine diphosphate, atropine sulphate, propranolol hydrochloride and glibenclamide from Sigma Aldrich (Milan, Italy).

\section{Results}

The mean physical characteristics of the 102 muscle strips from 29 animals (animals used for the effects of indomethacin and wash-out of PIN not included) were not significantly different between the matched experimental groups. The mean weight $( \pm \mathrm{SD})$ was $21 \pm$ $7 \mathrm{mg}$, the mean resting force $1 \pm 1 \mathrm{~g}$, and the mean maximal force $24 \pm 7 \mathrm{~g}$; maximal force was defined as maximum response to $10^{-4} \mathrm{M}$ ACh $(n=14)$, or $10^{-4} \mathrm{M}$ histamine $(n=8)$, or EFS at $25 \mathrm{~Hz}(n=7)$. The inhibitory effect of PIN $10^{-5} \mathrm{M}$ on contractions induced by EFS $(0.5 \mathrm{~ms}$ pulse duration, $25 \mathrm{~Hz}$, and $25 \mathrm{~V})$ developed slowly. There was a $40 \%$ drop in the force of contraction between 0 and $60 \mathrm{~min}$, with little change thereafter (fig. 1). The effect of PIN $10^{-5} \mathrm{M}$ was reversed after 120 min by repeated wash-outs with PSS at 5 min intervals. Incubation of muscles with $10^{-5} \mathrm{M}$ indomethacin did not significantly $(\mathrm{p}=0.67)$ alter the attenuation of the contractile response to EFS by PIN.

\section{Effect on EFS- and ACh-induced contractions}

Forty six strips from six animals were included in this part of the study. In one animal, no data for ACh-induced

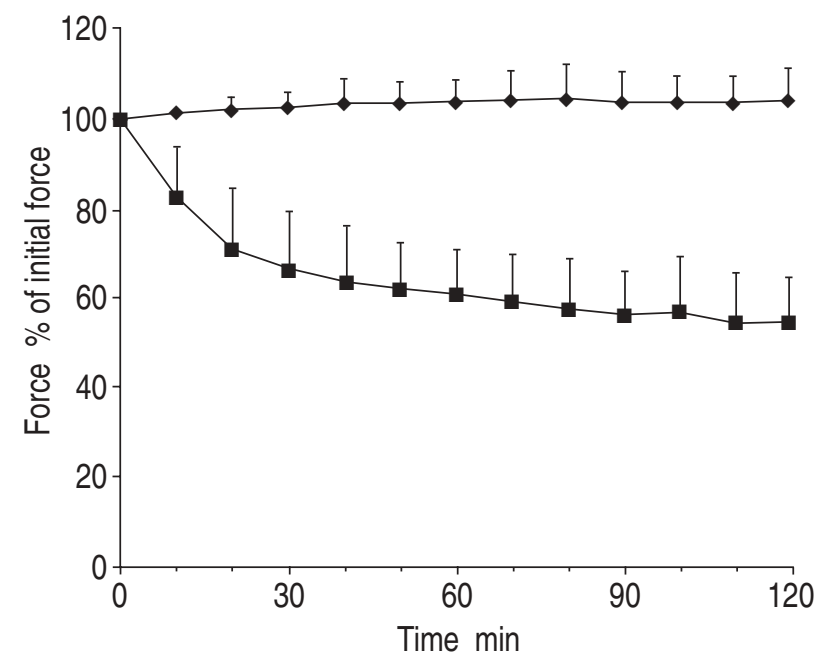

Fig. 1. - Effect of pinacidil on contractions induced by electric field stimulation $(0.5 \mathrm{~ms}$ pulse duration, $25 \mathrm{~Hz}$ and $25 \mathrm{~V})$. At time 0 , one strip (- ) from pairs of isolated bovine trachealis from seven animals was incubated with $10^{-5} \mathrm{M}$ pinacidil; the other strip (- - ) of the pair served as a control for the effect of time. Values are presented as mean $\pm \mathrm{SD}$ contractions could be obtained at $10^{-6} \mathrm{M}$ PIN. Since no paired data were available, data from this animal for this concentration were not included.

PIN $\left(10^{-5}-10^{-7} \mathrm{M}\right)$ had no significant effect on the resting forces $(\mathrm{p}>0.09)$. PIN $10^{-5}$ and $10^{-6} \mathrm{M}$ shifted the concentration-response curves to $\mathrm{ACh}(\mathrm{p}=0.0002$ and $\mathrm{p}=0.01$, respectively) and the frequency-response curves to EFS ( $p=0.003$ and $p=0.006$, respectively) significantly to the right; PIN $10^{-7} \mathrm{M}$ had no significant effect ( $\mathrm{p}=0.41$ and $\mathrm{p}=0.65$, respectively) (fig. 2).

To determine whether PIN had a prejunctional, postjunctional, or combined effect, its inhibitory effects on the ACh- and EFS-induced contractions were compared at the concentrations of $\mathrm{ACh}$ and frequencies of EFS resulting in 50 and $30 \%$ of the maximal response. There were no statistically significant differences $(\mathrm{p}=0.11)$ in the inhibitory effect of PIN $\left(10^{-5}-10^{-7} \mathrm{M}\right)$ (table 1$)$.

\section{Effect of glibenclamide}

The inhibitory effect of PIN $10^{-6} \mathrm{M}$ on both frequencyresponse to EFS and concentration-response to ACh was antagonized by glibenclamide in a concentration-dependent manner (fig. 3). Addition of glibenclamide $10^{-7} \mathrm{M}$ to strips incubated with PIN $10^{-6} \mathrm{M}$ caused a significant leftward shift of the frequency-response to EFS ( $p=$ $0.006)$ and the concentration-response to ACh $(\mathrm{p}=0.013)$. Glibenclamide $10^{-6}-10^{-5} \mathrm{M}$ completely antagonized the effect of PIN $10^{-6} \mathrm{M}$ on the frequency-response to EFS and the concentration-response to ACh.

\section{Effect on iNANC}

Eight trachealis strips from eight animals were precontracted to a force of $27 \pm 7 \mathrm{~g}$ with $10^{-4} \mathrm{M}$ histamine. Eight paired strips from the same eight animals were incubated with PIN $10^{-6} \mathrm{M}$ and then precontracted to a force of $28 \pm 5 \mathrm{~g}$ with $10^{-4} \mathrm{M}$ histamine. There was a significant frequency-dependent relaxation in response to EFS $(p<0.00001)$, but PIN $10^{-6} \mathrm{M}$ had no significant ( $p>0.99$ ) effect on the EFS-induced relaxations (fig. 4).

\section{Discussion}

The most important findings of this study on isolated bovine trachealis are that: 1) the inhibitory response to PIN developed gradually over a $1 \mathrm{~h}$ period; 2) PIN $10^{-5}$ $\mathrm{M}$ and $10^{-6} \mathrm{M}$, but not $10^{-7} \mathrm{M}$, significantly attenuated contractile responses to EFS and ACh; 3) glibenclamide antagonized the effect of PIN; 4) only a significant postjunctional mechanism of PIN was demonstrated; and 5) PIN $10^{-6} \mathrm{M}$ had no effect on iNANC nerves.

\section{Limitations}

Only isolated tissue was studied, hence the modulating effects of hormones and humoral mediators cannot be evaluated. In vivo, isotonic forces are required to alter airway calibre, but only isometric forces were measured in this study. It appears that either method results 

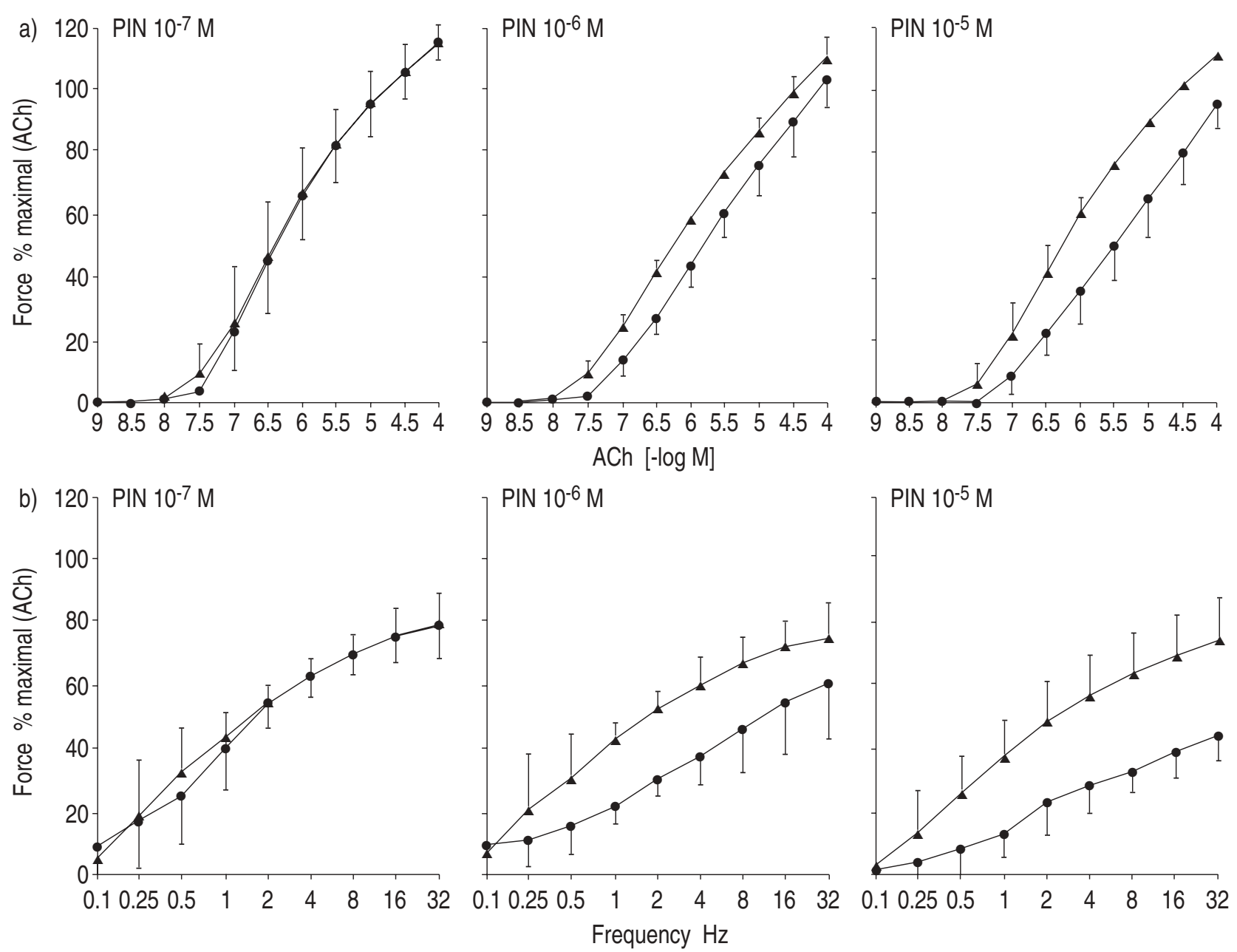

Fig. 2. - The effect of pinacidil (PIN): a) on cumulative concentration-response to ACh; and b) on frequency-response to EFS expressed as a $\%$ maximal force to $10^{-4} \mathrm{M}$ ACh. PIN $10^{-7} \mathrm{M}$ had no significant effect on either the frequency-response curve ( $\mathrm{p}=0.65$ ) or on the concentrationresponse curve $(\mathrm{p}=0.41)$. PIN $10^{-6} \mathrm{M}$ shifted both the frequency-response and the concentration-response curves significantly ( $\mathrm{p}=0.006$ and $\mathrm{p}=0.01$, respectively) to the right. Similarly, PIN $10^{-5} \mathrm{M}$ shifted both curves significantly ( $\mathrm{p}=0.003$ and $\mathrm{p}=0.0002$, respectively) to the right. $-\mathbf{\Delta}-$ : control, response of muscle before incubation with PIN; - - : response of the same muscle after incubation with PIN corrected for the effect of time. Values are presented as mean $\pm \mathrm{SD}(\mathrm{n}=6)$. ACh: acetylcholine; EFS: electrical field stimulation.

in similar conclusions [8]. Furthermore, only bovine tracheal muscles were used, i.e. one cannot exclude the possibility that peripheral bovine airways may respond differently to PIN than airways of other species, particularly those with eNANC nerves. Finally, tracheal muscles strips were denuded from epithelium, eliminating possible modulatory effects by the epithelium. Because of these limitations, one must be careful in

Table 1. - Inhibitory effect of pinacidil (PIN) on AChconcentration and EFS-frequency response

\begin{tabular}{lrrrrrr}
\hline & & \multicolumn{2}{c}{$\begin{array}{c}\text { Reduction of force } \\
\text { at } 30 \% \text { of response } \\
\text { PIN }\end{array}$} & & & \multicolumn{2}{c}{$\begin{array}{c}\text { Reduction of force } \\
\text { at } 50 \% \text { of response } \\
\text { to ACh } 10^{-4} \mathrm{M}\end{array}$} \\
\cline { 3 - 4 } \cline { 6 - 7 } & $\mathrm{n}$ & ACh & EFS & & ACh & EFS \\
\hline $10^{-7}$ & 6 & $3 \pm 2$ & $5 \pm 5$ & & $2 \pm 2$ & $3 \pm 2$ \\
$10^{-6}$ & 5 & $12 \pm 4$ & $15 \pm 7$ & & $15 \pm 4$ & $23 \pm 2$ \\
$10^{-5}$ & 6 & $17 \pm 3$ & $18 \pm 5$ & & $24 \pm 4$ & $26 \pm 4$ \\
\hline
\end{tabular}

Results are expressed as percentage of maximal response (ACh $10^{-4} \mathrm{M}$ ). Values are presented as mean $\pm \mathrm{SD}$. Responses to acetylcholine (ACh) and electrical field stimulation (EFS) were not significantly different $(\mathrm{p}=0.11)$. extrapolating the results to living subjects or other species.

\section{General comments}

KATP-channels are present in cell membranes of airway smooth muscles [9] and neurones [10]. The KATP-channels modulate contractile responses to various stimuli. A number of compounds have been developed for which the term potassium channel openers (KCOs) has been coined. These compounds include benzopyrene derivatives (cromakalim and its active enantiomer, lemakalim), cyanoguanide derivatives (pinacidil), and tetrahydrothiopyrens (RP 49356). Their main mechanism of action is thought to be an increase of $\mathrm{K}^{+}$efflux, thus leading to hyperpolarization of the cell, reduction of $\mathrm{Ca}^{2+}$-influx via L-type voltage-operated $\mathrm{Ca}^{2+}$-channels, and muscle relaxation. However, an effect on intracellular $\mathrm{Ca}^{2+}$ sequestration, reduction in smooth cell contractile properties, and protein kinase-A activation cannot be excluded. The relaxing effects of cromakalim, pinacidil, and RP 49356 are antagonized by glibenclamide, suggesting that KATPchannels are involved [11-13]. 

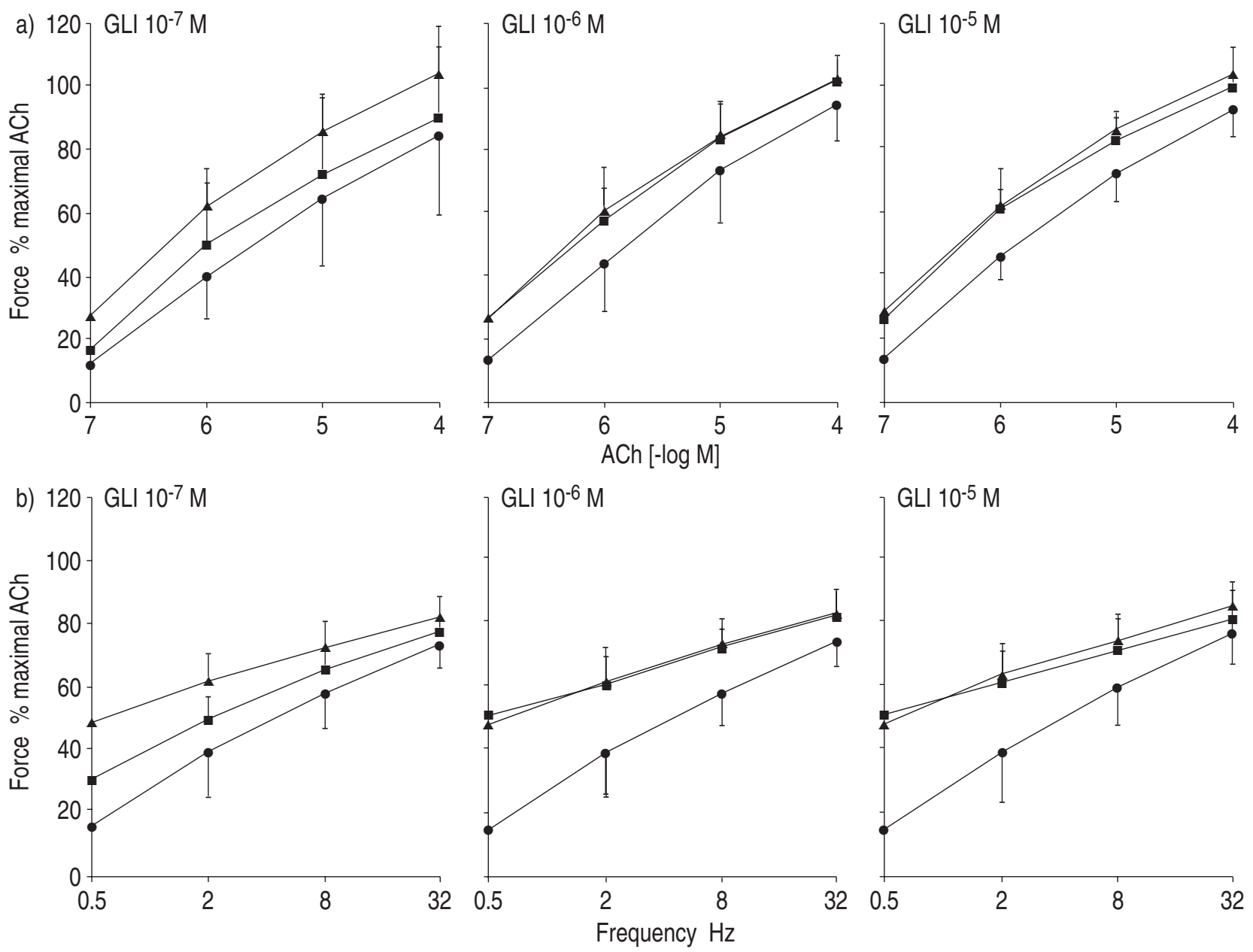

Fig. 3. - The effect of glibenclamide (GLI) a) on cumulative concentration-response to $\mathrm{ACh}$ and $\mathrm{b}$ ) on the frequency-response to EFS of bovine trachealis incubated with pinacidil (PIN) $10^{-6} \mathrm{M}$ expressed as a $\%$ maximal force to $10^{-4} \mathrm{M}$ ACh. The inhibitory effect of PIN both on AChand EFS-induced contractions was significantly attenuated by GLI $10^{-7} \mathrm{M}(\mathrm{p}=0.013$ and $\mathrm{p}=0.006)$ and completely abolished by GLI $10^{-6}$ and $10^{-5}$ M ( $>0.30$ vs control). - - - : control, response of muscle before incubation with PIN; $-\bullet$ : response of the same muscle after incubation with PIN corrected for the effect of time; —— : response of the same strips in the presence of PIN and glibenclamide. Values are presented as mean \pm SD $(n=4)$. ACh: acetylcholine; EFS: electrical field stimulation.

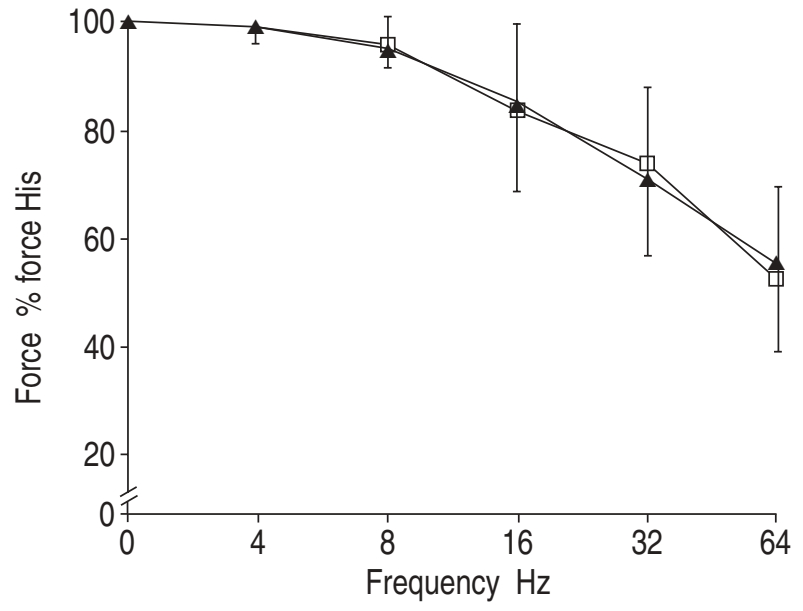

Fig. 4. - Relaxant effects of EFS on isolated bovine trachealis incubated with propranolol $10^{-6} \mathrm{M}$ and atropine $10^{-5} \mathrm{M}$ precontracted with histamine (His) $10^{-4} \mathrm{M}$ expressed as \% force induced by His. - $-\mathbf{\Delta}$ : muscle not incubated with pinacidil (PIN); —-: muscle incubated with PIN $10^{-6} \mathrm{M}$. Note the significant frequency-dependent relaxation $(\mathrm{p}<0.00001)$ and the absence of a significant effect of PIN $10^{-6} \mathrm{M}$ ( $>0.99)$, suggesting no effect on the inhibitory nonadrenergic-noncholinergic nervous system. Values are presented as mean \pm SD $(n=8)$. EFS: electrical field stimulation.

\section{Effect on EFS- and ACh-induced contractions}

EFS contracts airway smooth muscle by a neurallymediated (i.e. prejunctional) mechanism, and exogenous ACh by acting directly on airway smooth muscle (i.e. at postjunctional level). Therefore, comparisons of the effect of PIN on the ACh- and EFS response allow differentiation between pre- and postjunctional effects of PIN. As contractions induced by EFS and ACh may differ substantially, depending on the spreading of the stimulus through gap junctions and the number of muscarinic receptors occupied by exogenous ACh [14], the effects of PIN on contractions of the same absolute magnitude were compared. Although conclusive evidence for the lack of prejunctional effect of PIN can be obtained only by direct measurements of $\mathrm{ACh}$ release, the similarity of inhibitory effects of PIN $10^{-5}$ and $10^{-6} \mathrm{M}$ on EFSand ACh-induced contractions of the same magnitude strongly suggests a postjunctional effect in the bovine trachealis. This does not preclude the possibility that PIN may have an additional prejunctional effect in species with an eNANC nervous system.

Glibenclamide antagonized the effect of PIN, suggesting that bovine trachealis possesses KATP-channels. 
Furthermore, the similarity of the effect of glibenclamide on frequency-response to EFS and concentration-response to ACh suggests that the KATP-channels involved in the inhibitory effect of PIN on bovine trachealis contraction are located mainly at a postjunctional level.

Previous studies on the site of action of the KCOs have produced conflicting results. MCCAIG and DE JONCKHEERE [3] reported that cromakalim attenuated contractions induced by peripheral vagus nerve stimulation in the isolated guinea-pig trachea, but it had no effect on contractions induced by EFS or exogenous ACh, suggesting a ganglionic effect [3]. By contrast, BURKA et al. [4] and GOOD et al. [5] found in isolated guinea-pig trachea that cromakalim [4] or lemakalim [5] inhibited EFSinduced contractions more than ACh-induced contractions, suggesting pre- and postjunctional effects. Consistent with the latter results is the observation made in vivo by ICHINOSE and BARNES [6] that cromakalim inhibited bronchoconstriction induced by vagal stimulation more than that induced by exogenous ACh. In none of these studies, however, were the effects of KCOs compared on EFS- and ACh-induced contractions of the same magnitude.

There are several possible explanations of the difference between the present results and those of other studies. Firstly, PIN may possess a mode of activity different from that of cromakalim or lemakalim. This possibility was not investigated. Both compounds relax bovine trachealis by opening of rubidium-permeable, glibenclamide-sensitive KATP-channels [12]. However, the time for PIN to achieve a maximal effect was longer than that reported for cromakalim $[2,6]$, raising the possibility of different underlying mechanisms for relaxation. Further investigations are needed to determine the cause of the slow effect of PIN.

Secondly, the difference in the innervation of airways between species may be important. All of the studies cited above suggesting either prejunctional or pre- and postjunctional effects for the KOCs used tissue from guinea-pigs, a species possessing eNANC nerves in the airways. KCOs, including PIN, suppress the contractile responses evoked by eNANC stimulation but not by exogenous substance $\mathrm{P}[4,5]$, indicating that KOCs inhibit eNANC neuromodulation. As eNANC nerves seem to facilitate cholinergic neurotransmission [5], the much greater effect of cromakalim on EFS-induced contraction than on exogenous ACh-induced contraction could be attributed to a reduced cholinergic neurotransmission induced by a reduced eNANC activity. Bovine trachealis does not possess an eNANC system, as proved by the absence of any measurable EFS-induced contraction in the presence of atropine (data not shown), which makes EFS in this species a pure cholinergic excitatory stimulus. The lack of eNANC nerves in bovine trachea may explain the discrepancy between the present results and those of BuRKa et al. [4] and Good et al. [5], who found a prejunctional inhibitory effect of KCOs on eNANC responses in isolated guinea-pig trachealis. ICHINOSE and BARNES [6] found that cromakalim had a prejunctional inhibitory effect even in airways of guinea-pigs pre-treated with capsaicin, thus depleted of tachykinins, the putative eNANC mediators. Therefore, the absence of eNANC nerves in bovine trachealis cannot explain why it was not possible to demonstrate a prejunctional inhibitory effect of PIN on cholinergic neurotransmission in the present study.

Bovine tracheal smooth muscle does possess an iNANC system (fig. 4). If PIN inhibits iNANC nerves, its inhibitory effect on cholinergic neurotransmission may be underestimated. This possibility is unlikely, since PIN had no significant effect on the iNANC-mediated relaxation of trachealis precontracted with histamine. It is interesting to note that cromakalim also has no effect on iNANC-mediated relaxation of isolated guinea-pig trachealis [4].

The present results, suggesting a postjunctional site of action for KCOs, are consistent with recent results showing that neither cromakalim in isolated rat trachea [15] nor lemakalim in isolated guinea-pig trachea [16] inhibit electrically-evoked ACh release. In addition, BUCKLE et al. [17] found that PIN was able to relax human bronchi and guinea-pig trachealis contracted with histamine, suggesting a direct activity on airway smooth muscle.

In conclusion, the results of this study show that pinacidil attenuates the contraction of isolated bovine trachealis by acting at a postjunctional level. No significant effect on prejunctional cholinergic nerves could be demonstrated. This observation may be of clinical interest. Human airways, like bovine airways, have predominantly excitatory cholinergic and inhibitory nonadrenergic-noncholinergic nerves, hence the effect of pinacidil in humans may also be only postjunctional. Further investigations on acetylcholine release are necessary to confirm the absence of action of pinacidil and other potassium channel openers at prejunctional site.

\begin{abstract}
Acknowledgement: The authors appreciate the generosity of Formenti SpA (Milan, Italy) for providing pinacidil.
\end{abstract}

\section{References}

1. Cook NS. The pharmacology of potassium channels and their therapeutic potential. Trends Pharmacol Sci 1988; 9: 21-28.

2. Longmore J, Bray KM, Weston AH. The contribution of $\mathrm{Rb}$-permeable potassium channel to the relaxant and membrane hyperpolarizing actions of cromakalim, RP49356 and diazoxide in bovine tracheal smooth muscle. Br J Pharmacol 1991; 102: 979-985.

3. McCaig DJ, De Jonckheere B. Effect of cromakalim on bronchoconstriction evoked by cholinergic nerve stimulation in guinea-pig isolated trachea. Br J Pharmacol 1989; 98: 662-668.

4. Burka JF, Berry JL, Foster RW, Small RC, Watt AJ. Effects of cromakalim on neurally-mediated responses of guinea-pig tracheal smooth muscle. BrJ Pharmacol 1991; 104: 263-269.

5. Good DM, Clapham JC, Hamilton TC. Effects of BRL 38227 on neurally-mediated responses in the guineapig isolated bronchus. Br J Pharmacol 1992; 105: 933940.

6. Ichinose M, Barnes PJ. A potassium channel activator modulates both excitatory noncholinergic and cholinergic neurotransmission in guinea-pig airways. J Pharmacol Exp Ther 1990; 252: 1207-1212. 
7. Bai A, Eidelman DH, Hogg JG, et al. Proposed nomenclature for quantifying subdivisions of the bronchial wall. J Appl Physiol 1994; 77: 1011-1014.

8. Goldie RG. Evaluation of smooth muscle in vitro. In: Agrawal DK, Townley RG, eds. Airway Smooth Muscle: Modulation of Receptors and Response. Ann Arbor, Boston, CRC Press, 1990; pp. 115-117.

9. Collier ML, Twort CHC, Cameron IR, Ward JPT. BRL 38227 and ATP-dependent potassium channels in airway smooth muscle cells. In: Proceedings of New Drugs for Asthma. Official Satellite Symposium of the XI International Congress of Pharmacology. Davos, Switzerland, 1990; Abstract P4.

10. Hall AK, MacLagan J. Effect of cromakalim on cholinergic neurotransmission in the guinea-pig trachea. $\mathrm{Br}$ J Pharmacol 1988; 95: 792P.

11. Murray MA, Boyle JP, Small RC. Cromakalim-induced relaxation of guinea-pig isolated trachealis: antagonism by glibenclamide and by phentolamine. Br J Pharmacol 1989; 98: 865-874.

12. Gater PR. Effects of $\mathrm{K}^{+}$-channel openers on bovine tracheal smooth muscle. Br J Pharmacol 1989; 98: 660P.
13. Reaburn DM, Brown TJ. RP 49356 and cromakalim relax airway smooth muscle in vitro by opening a sulphonylurea-sensitive $\mathrm{K}^{+}$-channel: a comparison with nifedipine. J Pharmacol Exp Ther 1991; 256: 492-499.

14. Daniel EE, Kannan M, Davis C, Posey-Daniel V. Ultrastructural studies on the neuromuscular control of human tracheal and bronchial muscle. Respir Physiol 1986; 63: 109-128.

15. Wessler I, Holz C, MacIagan J, Pohan D, Reinheimer T, Racké K. Cromakalim inhibits electrically-evoked $\left[{ }^{3} \mathrm{H}\right]$ acetylcholine release from a tube-preparation of the rat isolated trachea by an epithelium-dependent mechanism. Naunyn Sch Arch Pharmacol 1993; 348: 14-20.

16. Baker DG, Don HF, Brown JK. Pharmacologic activation of $\mathrm{K}^{+}$-channels does not inhibit $\mathrm{Ca}^{2+}$-dependent, stimulus-evoked release of acetylcholine (ACh) in guineapig trachea. Am Rev Respir Dis 1993; 147: A504.

17. Buckle DR, Arch JRS, Bowring NE, et al. Relaxant effects of the potassium channel activators BRL 38227 and pinacidil on guinea-pig and human airway smooth muscle, and blockade of their effects by glibenclamide and BRL 31660. Pulm Pharmacol 1993; 6: 77-86. 